ductal carcinoma; 2) high-grade intraepithelial neoplasia (HGPIN); 3) intraductal carcinoma; and, 4) intraductal spread of an invasive carcinoma.

Ductal carcinoma may be ruled out because of the presence of basal cells; in cases of irregularity and distortion of the ducts, HGPIN may also be ruled out; and, in presence of an invasive carcinoma, intraductal spread is most probable and the finding adds no additional information to the report. The problem is related to cases that IDC-P is the only finding on a biopsy.

The Johns Hopkins group studied 20 radical prostatectomy specimens of patients who presented on biopsy only the diagnosis of IDC-P. Two out of twenty patients showed extensive IDC-P only, without identifiable invasive cancer. IDC-P in these 2 patients may represent: 1 . an early phase of ductal or acinar carcinoma; or 2. intraductal spread of an invasive not detected tumor.

According to the authors, IDC-P as the only finding on needle biopsy corresponds to almost always to aggressive tumors and the patients may have definitive treatment. A more conservative approach would be an extended rebiopsy in order to detect a possible invasive cancer.

\title{
References
}

1. Cohen RJ, McNeal JE, Baillie T: Patterns of differentiation and proliferation in intraductal carcinoma of the prostate: significance for cancer progression. Prostate. 2000; 43: 11-9.

2. Guo CC, Epstein JI: Intraductal carcinoma of the prostate on needle biopsy: Histologic features and clinical significance. Mod Pathol. 2006; 19: 1528-35.

\author{
Dr. Athanase Billis \\ Full-Professor of Pathology \\ State University of Campinas, Unicamp \\ Campinas, São Paulo, Brazil \\ E-mail: athanase@fcm.unicamp.br
}

\section{BASIC AND TRANSLATIONAL UROLOGY}

\section{Potential mechanism of action of human growth hormone on isolated human penile erectile tissue}

Uckert S, Scheller F, Stief CG, Knapp WH, Sohn M, Becker AJ, Kuczyk MA

Department of Urology and Uro-Oncology, Hannover Medical School, Hannover, Germany

Urology. 2010; 75: 968-73

Objectives: To evaluate the mechanisms of growth hormone $(\mathrm{GH})$ action on isolated human penile erectile tissue. Human $\mathrm{GH}(\mathrm{hGH})$ has been suggested to play a role in male reproductive function, including penile erection. Nevertheless, it still remains unclear which intracellular pathways mediate the physiological effects of $\mathrm{GH}$ on the human corpus cavernosum (HCC).

Methods: Using the organ bath technique, the effects of GH were investigated on electrical field stimulation (EFS)-induced relaxation of isolated $\mathrm{HCC}$ in the absence and presence of the guanylyl cyclase inhibitor $1 \mathrm{H}-$ 
[1,2,4] oxadiazolo[4,3-a]quinoxalin-1-one (ODQ) and nitric oxide synthase (NOS) inhibitor N(G)-nitro-1-arginine (1-NOARG, 10 microm). Effects of GH on the production of tissue cyclic guanosine monophosphate (cGMP) in the absence and presence of ODQ and 1-NOARG were also elucidated using radioimmunoassay.

Results: ODQ and 1-NOARG abolished the relaxation of the tissue induced by EFS, whereas amplitudes were increased by physiological concentrations of GH $(1-100 \mathrm{~nm})$. The attenuation of EFS-induced amplitudes by 1-NOARG but not ODQ was, in part, reversed by GH. The production of cGMP (pmol cGMP/mg protein) induced by $10 \mathrm{~nm}$ GH was abolished in the presence of 10 microm ODQ. In contrast, the combination of GH $(10 \mathrm{~nm})$ and 1-NOARG (10 microm) maintained cGMP production significantly greater than baseline $(0.68$ +/- 0.36 vs 1.07 +/- $0.48 \mathrm{pmol} \mathrm{cGMP} / \mathrm{mg}$ protein).

Conclusions: Our data provide evidence that GH may act on human HCC by an NO-independent effect on guanylyl cyclase activity and may thus explain how growth factors, such as hGH, regulate male erectile function. Copyright 2010 Elsevier Inc. All rights reserved.

\title{
Editorial Comment
}

This group of investigators has been studying the effects of growth hormone for more than 10 years. They have demonstrated previously that growth hormone likely mediates penile erection through its stimulating effect on the cGMP pathway in human cavernous smooth muscle. Later, they compared the in vivo serum profiles of growth hormone in the systemic and cavernous blood samples obtained from healthy volunteers were compared to the serum profiles of patients with erectile dysfunction. In the healthy subjects, systemic growth hormone serum levels significantly increased during penile tumescence, followed by a transient decline from tumescence to rigidity and detumescence. During penile tumescence, the mean increase in the growth hormone levels in the systemic and cavernous blood of patients with organogenic dysfunction, this increase was found to be negligible.

In the present study they found evidence that growth hormone may act on human corpus cavernosum by an effect independent of nitric oxide on guanylyl cyclase activity. The group has to be commended for the important contribution they have been providing during the last years to elucidation of growth hormone activity in human erection

\author{
Dr. Francisco J. B. Sampaio \\ Full-Professor and Chair, Urogenital Research Unit \\ State University of Rio de Janeiro \\ Rio de Janeiro, RJ, Brazil \\ E-mail: sampaio@urogenitalresearch.org
}

doi: $10.1590 / \mathrm{S} 1677-553820100002000023$

\author{
Atorvastatin protects renal function in the rat with acute unilateral ureteral obstruction \\ Kamdar C, Chou SY, Mooppan UM, Kim H, Gulmi FA \\ Department of Urology, Brookdale University Hospital and Medical Center, Brooklyn, New York, USA \\ Urology. 2010; 75: 853-7
}

Objectives: To examine the effects of atorvastatin on renal hemodynamics and urinary microalbumin levels in rats with acute unilateral ureteral obstruction (UUO). Previous studies have demonstrated that treatment with statins attenuated renal structural damages in rodents with chronic UUO. However, it is not known whether statins afford protection of renal function. 